\title{
Energy-Aware Adaptive Cooperative FEC Protocol in MIMO Channel for Wireless Sensor Networks
}

\author{
Yong Jin $^{1}$ and Guangwei Bai ${ }^{2}$ \\ ${ }^{1}$ School of Computer Science \& Engineering, Changshu Institute of Technology, Changshu 215500, China \\ ${ }^{2}$ Department of Computer Science \& Technology, Nanjing University of Technology, Nanjing 211816, China
}

Correspondence should be addressed to Yong Jin; njutjinyong@yahoo.com.cn

Received 26 March 2013; Accepted 23 June 2013

Academic Editor: Dharma Agrawal

Copyright (C) 2013 Y. Jin and G. Bai. This is an open access article distributed under the Creative Commons Attribution License, which permits unrestricted use, distribution, and reproduction in any medium, provided the original work is properly cited.

\begin{abstract}
We propose an adaptive cooperative forward error correction (ACFEC) based on energy efficiency combining Reed-Solomon (RS) coder algorithm and multiple input multiple output (MIMO) channel technology with monitoring signal-to-noise ratio (SNR) in wireless sensor networks. First, we propose a new Markov chain model for FEC based on RS codes and derive the expressions for QoS on the basis of this model, which comprise four metrics: throughput, packet error rate, delay, and energy efficiency. Then, we apply RS codes with the MIMO channel technology to the cross-layer design. Numerical and simulation results show that the joint design of MIMO and adaptive cooperative FEC based on RS codes can achieve considerable spectral efficiency gain, real-time performance, reliability, and energy utility.
\end{abstract}

\section{Introduction}

There is an unprecedented revolution in wireless sensor networks [1,2], which was driven by the explosive growth and diversity of wireless application services such as data acquisition and interactive mobile multimedia applications. Various sophisticated applications in wireless sensor networks require different quality of service (QoS) satisfaction. In order to prolong the lifetime of sensor network and achieve better utilization of scarce radio resources, the cooperative communication approach and error control schemes have drawn significant research attention [3-8].

The use of forward error correction (FEC) [9] scheme is a classical solution to improve the reliability of data transmissions in wireless sensor networks. Specially, packetbased Reed-Solomon coder [10] has been already used in many application services of wireless sensor networks. In particular, many of delay-sensitive multimedia services rely on the Reed-Solomon (RS) coder. An adaptive FEC technique was proposed in the literature [3], which dynamically tunes the amount of FEC code based on the arrival of acknowledgement packets and out of control with signalto-noise ratio (SNR) or bit error rate from receivers. Our previous study [4] discussed the energy efficiency of FEC on distance and predicts the frame loss rate with GM (1, 1) model, on the basis of adjusting the FEC parameter of sensor nodes. However, energy saving and consumption is a chief issue in wireless sensor networks. So, Gupta et al. [5] proposed an energy-efficient data gathering protocol that uses a prediction-based filtering mechanism to solve the problem of redundant data transmissions. At the same time, Singh et al. [6] proposed an approach of energy-efficient transmission error recovery algorithm. Furthermore, the multi-input multi-output (MIMO) scheme is usually used in wireless sensor networks in order to reduce the fading effects in wireless channel [11]. A distributed threshold based on MAC protocol for cooperative MIMO transmissions was proposed in the literature [7] which uses a threshold scheme based on the queue length and minimizes latency ensuring the stability of transmission queues. A clustered wireless sensor networks were proposed by Del Coso et al. [8] for minimum end-to-end outage probability and presented a per-link energy constraint in cooperative distributed MIMO channels.

However, there are some drawbacks in all these methods and research findings explained above. The influence of RS coder on packet error rate and communication quality in cooperative transmission is not considered in $[3,4]$, which 
also ignore the combination of RS coder and MIMO channel technology. The energy information based on RS coder is not mentioned in $[5,6]$. How to select optimal relay nodes adaptively and set up the parameters of FEC schemes dynamically was not considered in $[7,8]$.

On the basis of the above researches, we analyze the adaptive cooperative FEC mechanism, in the hope that the data could be transmitted in wireless sensor networks with high quality. First of all, we combine the RS coder and MIMO channel technology. The relay node could be chosen according to the SNR threshold based on the characteristics of energy efficiency secondly. Our analytical and simulation results show that our proposed mechanism is capable of utilizing the available network resource and achieve good perceptual quality, in terms of system throughput, reliability, and real-time performance, as well as maximization of the wireless sensor networks lifetime.

\section{System Model}

2.1. Network Model. The FEC system model based on RS codes in MIMO channels using STBCs of wireless senor networks is shown in Figure 1. Assume that there are $N_{t}$ transmit antennas and $N_{r}$ receive antennas.

The multiple modulation and coding schemes are used at the physical layer. The symbols are coded and transmitted via the MIMO fading channels after space-time block coding. The SNR is monitored at the receiver node and then sent back through a feedback channel to the FEC controller, which chooses the appropriate parameters of RS coder in the next transmission accordingly.

At the data link layer, the FEC protocol is adopted to error control of packet transmissions. The raw data packet is encoded by the $\operatorname{RS}(n, k)$ with $m$ encoder. Here, $m$ is the length of the elements in the finite field, which is in bits and belongs to $\{2 \cdots 16\}$. Note that the number of data packets generated from the source packets is denoted by $n$ and the number of redundant data packets which are made from $k$ raw data packets is denoted by $n-k$ and is the total number of data packets. In particular $n$ and $k$ must be differing by a positive even integer; $n$ belongs to the extent of $\left[k, 2^{m}\right]$. When an error is detected in a packet, $k$ data packets can be recovered according to any $k$ or more data packets which are correctly received by the receiver node. Let $P_{\mathrm{FEC}}$ denote the packet error rate of all the data packets, which is given by (1)

$$
P_{\mathrm{FEC}}=1-\sum_{l=k}^{n}\left(\begin{array}{l}
n \\
l
\end{array}\right)(1-p)^{l} p^{n-l}
$$

where the average packet error rate of system is denoted by $p$. If the error is not corrected successfully in a packet, a retransmission request is generated and sent back to the transmitter through the MIMO feedback channel. Therefore, we describe the wireless network condition with five parameters, which are $\left\langle m, n, k, N_{t}, N_{r}\right\rangle$.

2.2. Markov Chain Model. For studying the transitions process between different sensor nodes in wireless sensor networks exactly and conveniently, we proposed a new Markov chain model for FEC scheme after considering the proposed Markov model for reliable packet delivery in the literature [12]. Let $H(t)$ denote the length of elements in the finite field and let $F(t)$ denote the number of data packets generated from the source packets of RS coder in sensor node at $t$ time. Because the outage of wireless channel, packet error and overtime are the independent stochastic process each other, the future outage of wireless link has nothing to do with the past damaged packets. Hence, the bidimensional random process of $\{H(t), F(t)\}$ is discrete-time Markov chain depicted in Figure 2. In this Markov chain, the one-step transition probabilities are

$$
\begin{aligned}
& P\{m, n \mid m, n+1\}=1-P_{\mathrm{FEC}}, \\
& P\{m+1, n \mid m, n\}=P_{\mathrm{FEC} .}^{n}
\end{aligned}
$$

Here, note that $P\left\{m_{2}, n_{2} \mid m_{1}, n_{1}\right\}=P\left\{H(t)=m_{2}, F(t)=\right.$ $\left.n_{2} \mid H(t)=m_{0}, F(t)=n_{0}\right\}$. As shown in Figure 2, when the values of $m$ and $n$ are lager than $m_{\max }$ and $n_{\max }$, respectively, at the same time, the data packet would be dropped by the sender node. So, let $P_{\mathrm{AC}}$ denote the packet dropping rate. Then, let $b_{j, k}=\lim _{t \rightarrow \infty} P\{H(t)=m, F(t)=n\}$ be the stationary distribution of this Markov chain. Hence, the closed-form solution is given by (3)

$$
\begin{gathered}
b_{m, 1}=P_{\mathrm{FEC}} b_{1,1} \quad m \in\left[2, m_{\max }\right] \\
b_{m, n}=\left(\frac{m-k}{m}\right)\left(\frac{1}{1-P_{\mathrm{FEC}}}\right) \quad m \in\left[2, m_{\max }\right] \\
\sum_{m=2}^{m_{\max }} \sum_{n=1}^{n_{\max }} b_{m, n}=1
\end{gathered}
$$

where $b_{1,1}$ is the stationary probability when $m$ is 2 and $n$ is 1 and $b_{m, 1}$ is the stationary probability when $m$ is $2^{n}$ and $n$ is 1 . According to (3), we can have the following equation:

$$
b_{1,1}=\frac{1}{\sum_{m=2}^{m_{\max }}\left(1+\left(1 /\left(1-P_{\mathrm{FEC}}\right)\right)(n / 2)\right) P_{\mathrm{AC}}^{n}} .
$$

The data packet would be dropped when $m$ is larger than $m_{\max }$ and $n$ is larger than $n_{\max }$ simultaneously, which is given by

$$
P_{\mathrm{AC}}=P_{\mathrm{FEC}}^{m_{\max }+n_{\max }} .
$$

When the physical characteristics of sensor node and $k$ of RS coder are known, $P_{\mathrm{FEC}}$ and $P_{\mathrm{AC}}$ can be calculated from (1) to (5), which are unique. Therefore, the analytical model of saturation throughput, average delay, and energy efficiency would be given and discussed in the next section.

2.3. QoS Analytical Model. At first, let $S_{T}$ denote the normalized saturation throughput, which is shown in

$$
S_{T}=\left(1-P_{\mathrm{FEC}}\right)\left(1-P_{\mathrm{FEC}}^{m_{\max }+1}\right)^{n}
$$

Secondly, saturation delay is the average delay under the saturation condition, which considers the modulation delay, 


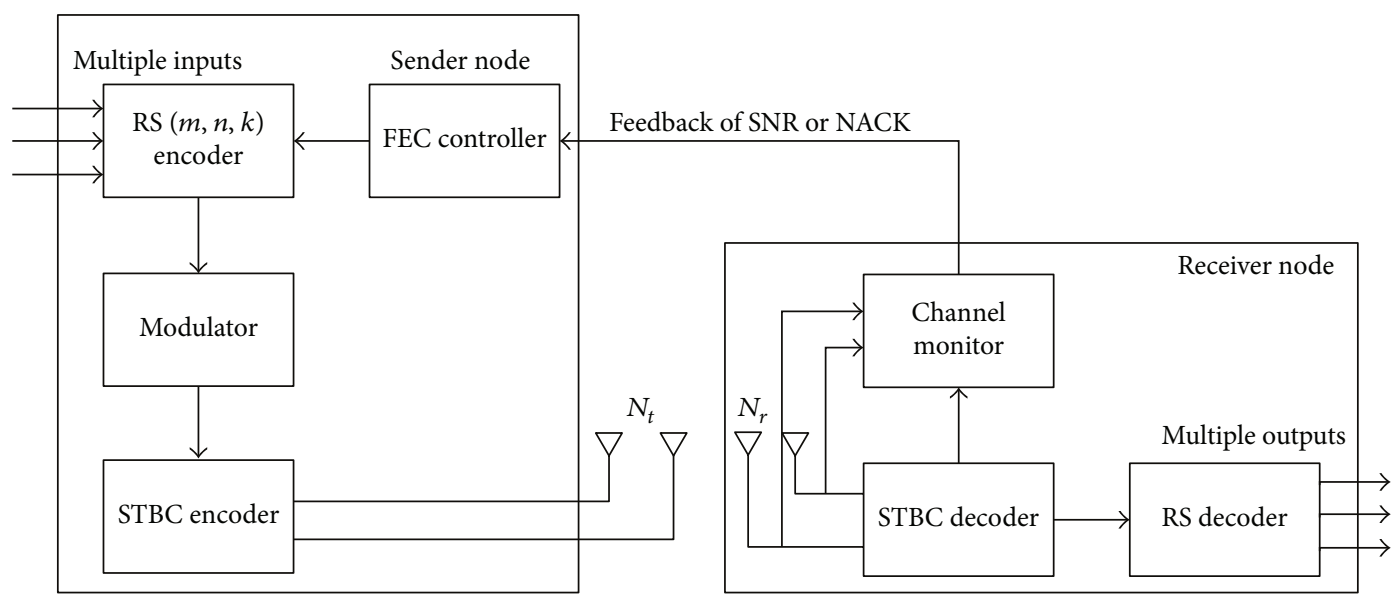

FIgURE 1: Network model of FEC-MIMO.

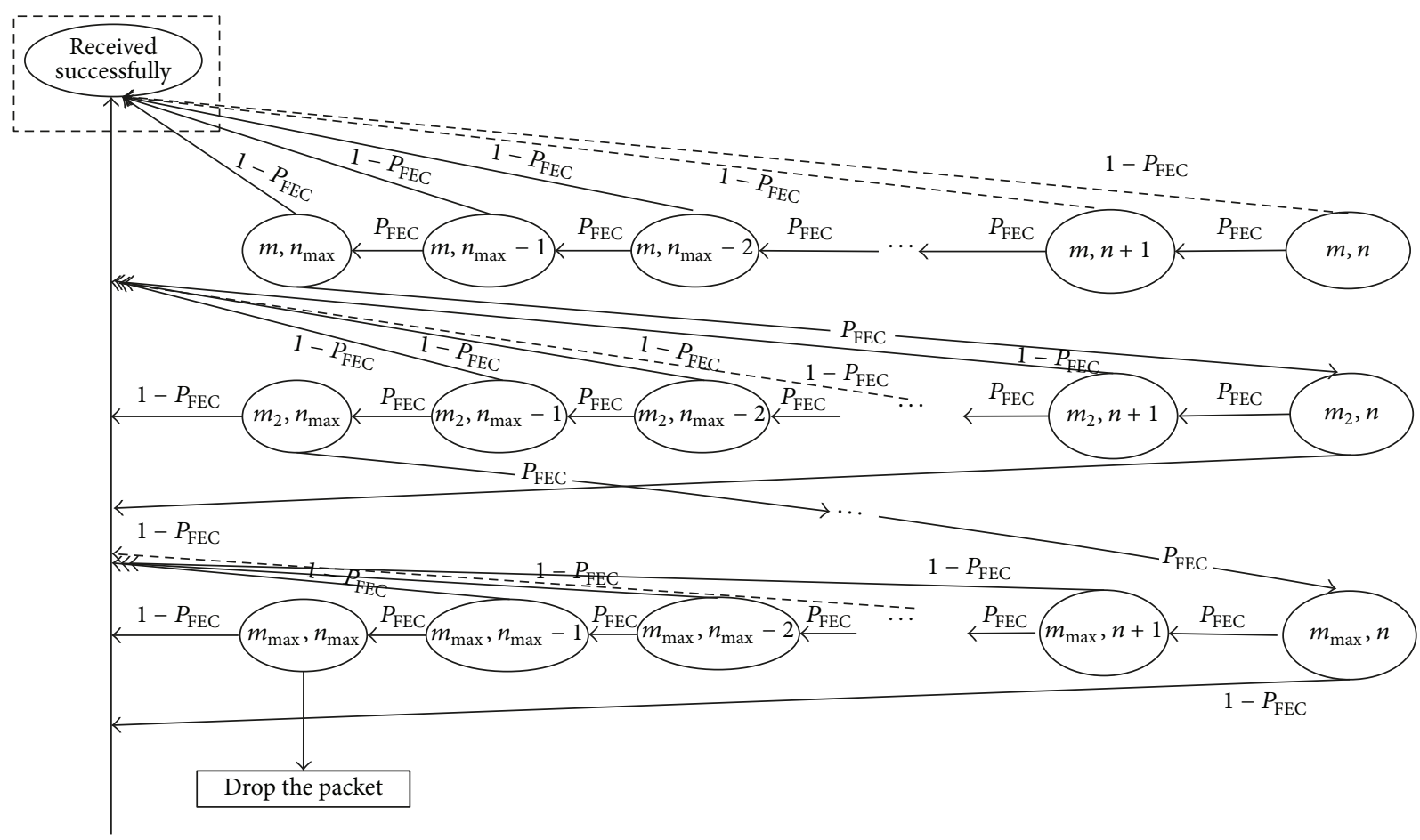

Figure 2: Markov chain model for FEC scheme.

RS encoder and decoder delay, and transmission delay, as well as being denoted by $T_{\mathrm{AC}}$ and is given by

$$
T_{\mathrm{AC}}=k T \frac{1-P_{\mathrm{FEC}}^{m_{\max }+1}}{1-p_{\mathrm{FEC}}} .
$$

Here, let $T$ denote the transmission delay of one packet at data link layer.

Finally, based on our previous study [4], the encoding energy consumption is much lower than decoding in RS coder, so we only consider the energy consumption of decoding. Besides, the energy consumption of starting a sensor, sending and receiving a packet are also taken into account (i.e., $E_{\text {start }}, E_{\text {tran }}^{\mathrm{FEC}}$, and $E_{\mathrm{re}}^{\mathrm{FEC}}$ ).
If the data length of transmitting is $L$ bits, the transmitting code number $C_{n}$ is given by

$$
C_{n}=\left\lceil\frac{L}{k}\right\rceil
$$

The energy consumption of RS $(n, k)$ decoder (i.e., $\left.E_{\mathrm{dec}}\right)$ is given by

$$
E_{\mathrm{dec}}=C_{n}\left(\left(4 n t+10 t^{2}\right) E_{\mathrm{mult}}+\left(4 n t+6 t^{2}\right) E_{\mathrm{add}}+3 t E_{\mathrm{inv}}\right) .
$$


TABLE 1: Definitions and value of parameters in (9) and (10).

\begin{tabular}{lcc}
\hline Parameters & Definition & Value \\
\hline$E_{\text {start }}$ & Starting energy consumption & $2.5 \mathrm{nj} / \mathrm{frame}$ \\
$E_{\text {add }}$ & Energy consumption of addition & $3.3 \times 10^{-5} \mathrm{~m}(\mathrm{~mW} / \mathrm{MHz})$ \\
$E_{\text {mult }}$ & Energy consumption of multiplication & $3.7 \times 10^{-5} \mathrm{~m}^{3}(\mathrm{~mW} / \mathrm{MHz})$ \\
$E_{\text {inv }}$ & Energy consumption of reciprocal in $\mathrm{GF}\left(2^{m}\right)$ field & $3.3 \times 10^{-5}(2 \mathrm{~m}-3) \mathrm{m}^{3}(\mathrm{~mW} / \mathrm{MHz})$ \\
$V_{\text {radio }}$ & Supply voltage for radio & $3 \mathrm{v}$ \\
$I_{\text {tr }}$ & Transmit current & $8.5 \mathrm{~mA}$ \\
$I_{\text {re }}$ & Receive current & $7 \mathrm{~mA}$ \\
$\alpha$ & Sum of header and FCS size & $11 \mathrm{bytes}$ \\
\hline
\end{tabular}

Here, $E_{\text {add }}, E_{\text {mult }}$, and $E_{\text {inv }}$, respectively, represent the energy consumption of addition, multiplication, and reciprocal in $\operatorname{GF}\left(2^{m}\right)$ field. So the total energy consumption of FEC is given by

$$
\eta_{\mathrm{AC}}=\frac{\left(\left(I_{\mathrm{tr}} I_{\mathrm{re}}\right) V_{\text {radio }}(\ln (k) / \log 2) T_{\mathrm{tr}}\right)\left(1-P_{\mathrm{FEC}}\right)}{E_{\text {start }}+\left(I_{\mathrm{tr}} I_{\mathrm{re}}\right) V_{\text {radio }}(\log n / \log 2+\alpha) T_{\mathrm{tr}}+E_{\mathrm{dec}}} .
$$

Note the parameter settings of (9) and (10) based on Mica2 sensor node, which are given in Table 1.

\section{Energy Aware Adaptive Cooperative FEC}

3.1. Relay Selection Protocol. We discover that the energy efficiency of FEC scheme has close relationship with SNR and $m$ at first. Then, the variation characteristic of this scheme energy efficiency is presented by mathematical analyses in different SNR. The change trend of energy efficiency as a function of SNR is shown in Figure 3. It was obvious that the energy efficiency maintained a steady upward trend with increasing SNR.

In particular, the energy efficiency increases to the maximum value rapidly when the SNR between sender node and next hop receiver node is greater than $13 \mathrm{~dB}$ using $\mathrm{RS}(13,9)$ with $m=4$. Hence, SNR between sender node and next hop receiver node has a constant value, which is $13 \mathrm{~dB}$ for this scheme. There is apparently an SNR threshold value based on energy efficiency of FEC scheme. Likewise, the SNR threshold values of different RS coders are shown in Figure 3(a) with $m=4$ and in Figure 3(b) with $m=6$. Therefore, different RS coder algorithms have always one constant and energyefficiency aware SNR threshold value. We can choose the best relay node as the next hop receiving node according to this conclusion.

In summary, if we can obtain the SNR of MIMO channel, the optimal relay node should be chosen according to SNR threshold. The process of relay selection is as follows.

(1) The SNR of MIMO channel is acquired with real-time detection.

(2) Gain the SNR threshold $\mathrm{SNR}_{\mathrm{TE}}$ based on the QoS analytical model.

(3) If the SNR of one sensor node is less than or equal to $\mathrm{SNR}_{\mathrm{TE}}$, it would be selected as the relay node.
3.2. Combination of RS Coder and MIMO Channel. In this subsection, we define five combination scenarios of RS coder and MIMO channel: (1) MIMO $(2,1),(2)$ MIMO $(2,1)$ and RS $(15,11)$ with $m=4,(3) \operatorname{MIMO}(2,2)$ and $\operatorname{RS}(15,11)$ with $m=4$, (4) MIMO $(2,1)$ and RS $(7,3)$ with $m=3$, and (5) MIMO (2, 2 ) and RS $(7,3)$ with $m=3$. On the basis of the QoS analysis model based on Markov chain, the variation trend of bit error rate with SNR is illustrated in Figure 4.

From Figure 4, we found the relationship of five combination schemes of RS coder and MIMO channel, which are listed as follows: $\operatorname{MIMO}(2,1)>\operatorname{MIMO}(2,1)$ and $\operatorname{RS}(15,11)$ with $m=4>\operatorname{MIMO}(2,2)$ and RS $(15,11)$ with $m=4>$ MIMO $(2,1)$ and RS $(7,3)$ with $m=3 \geq \operatorname{MIMO}(2,2)$ and RS $(7,3)$ with $m=3$.

We found out that the reliability of MIMO and RS coder is superior to one of MIMO channel alone. In particular, the bigger the value of $N_{t}$ transmit antennas and $N_{r}$ receive antennas, the better the reliability of wireless channel. Therefore, the sender and receiver nodes could select the optimal combination scheme to satisfy the diversity requirement of QoS.

3.3. Algorithm of Adaptive Cooperative FEC. The energyaware adaptive cooperative FEC mechanism (ACFEC) is proposed in this section, which is based on the combination of RS coder algorithm and MIMO channel. Because the relay node is selected based on characteristics of energy efficiency and SNR, the performance of the proposed mechanism can be evaluated by the following equation:

$$
\mathrm{Q}_{\mathrm{ACFEC}}=\sum_{m=1}^{m_{\max }} \mathrm{Q}_{\mathrm{AC}}\left(m, n_{\max }\right)+q,
$$

where $Q_{\text {ACFEC }}$ is the performance metrics of ACFEC, which include throughput ratio, packet error rate, average delay, and energy efficiency. Let $Q_{\mathrm{AC}}\left(m, n_{\max }\right)$ denote the above four performance metrics when SNR is greater than $\mathrm{SNR}_{\mathrm{TE}}$. Let $q$ record these metrics when $\mathrm{SNR}$ is less than $\mathrm{SNR}_{\mathrm{TE}}$.

Afterwards, we present the basic principle of the ACFEC and its implementation at sender node, receiver node, and relay nodes in detail, which is illustrated as follows.

\section{At Sender Node}

Step (1): carry out the combination scheme of RS coder and MIMO channel technology. The guarantee 


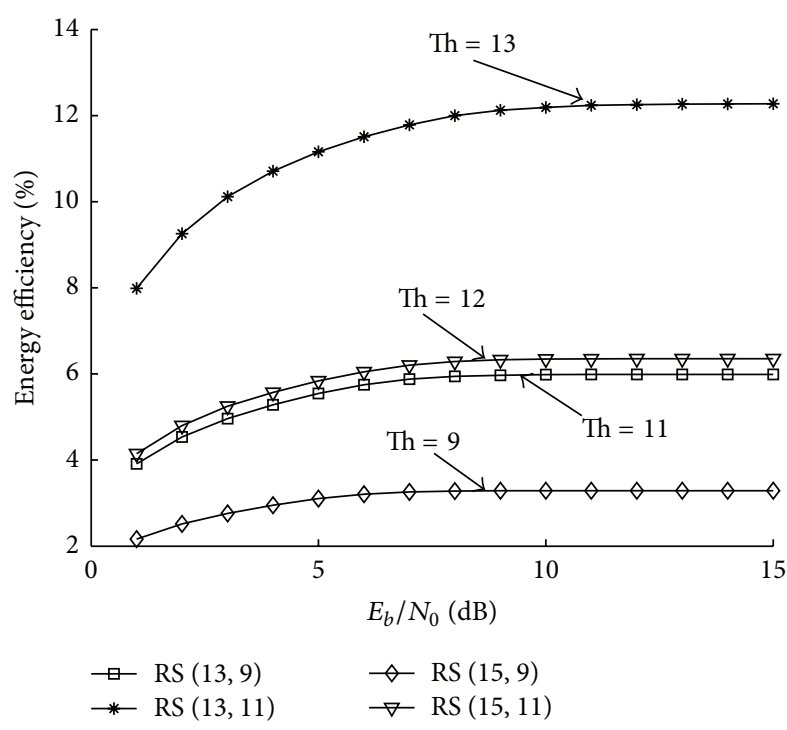

(a) $m=4$

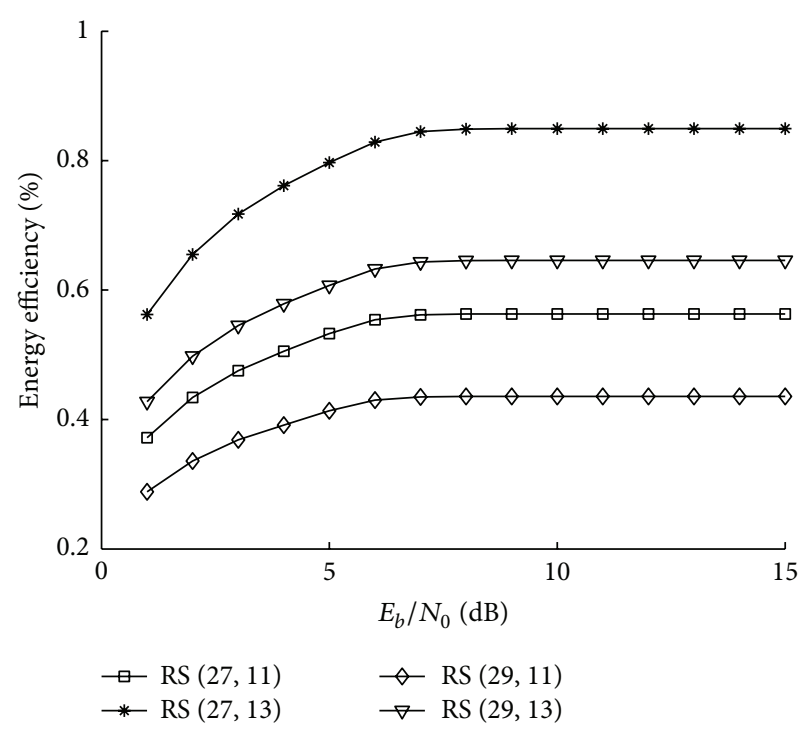

(b) $m=6$

FIGURE 3: Analytical research of energy efficiency along with SNR.

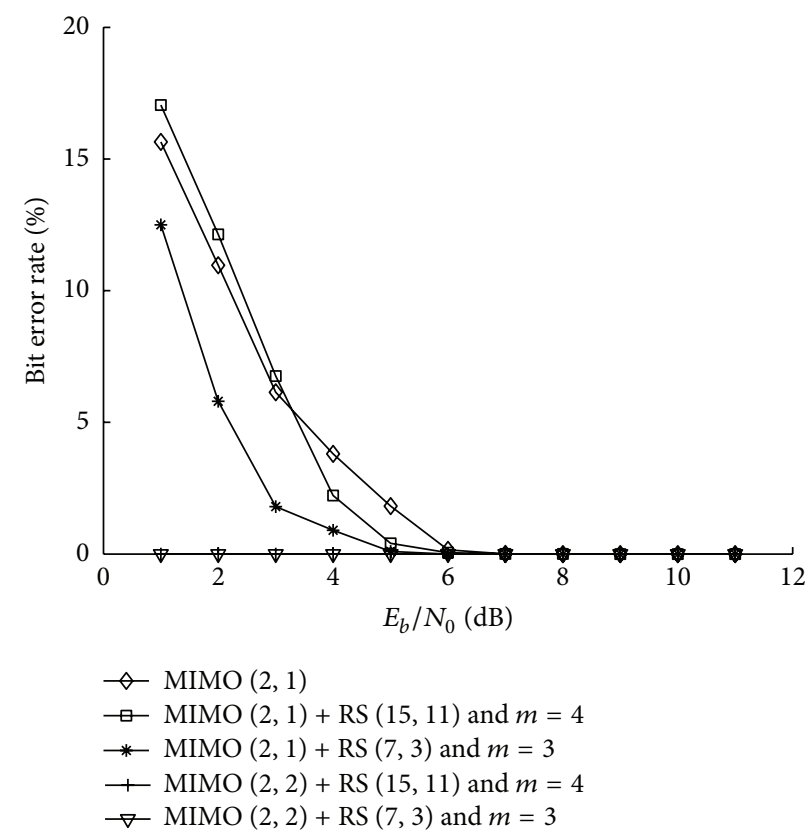

FIGURE 4: Performance comparisons between different combination scenarios of RS coder and MIMO channel.

priority of reliability is appointed according to the diversity requirement.

Step (2): initialize the network parameters: $m, n, k, N_{t}$, and $N_{r}$ on the basis of Step (1) results. FEC scheme based on RS coder is implemented. Moreover, the values of $\mathrm{SNR}_{\mathrm{TE}}$ and $m_{\max }$ are obtained based on the QoS analytical model.

Step (3): relay selection mechanism based on energy efficiency and SNR is implemented when channel state information is known.
Step (4): start to send data packets by means of MIMO $\left(N_{t}, N_{r}\right)$ channel.

Step (5): when the timer matures or NACK packet is received, go to Step (1).

The pseudocode for the proposed scheme at sender node is summarized in Algorithm 1.

\section{Relay Nodes}

Step (6): select the optimal relay node from candidate nodes according to energy-efficiency-aware SNR threshold. 


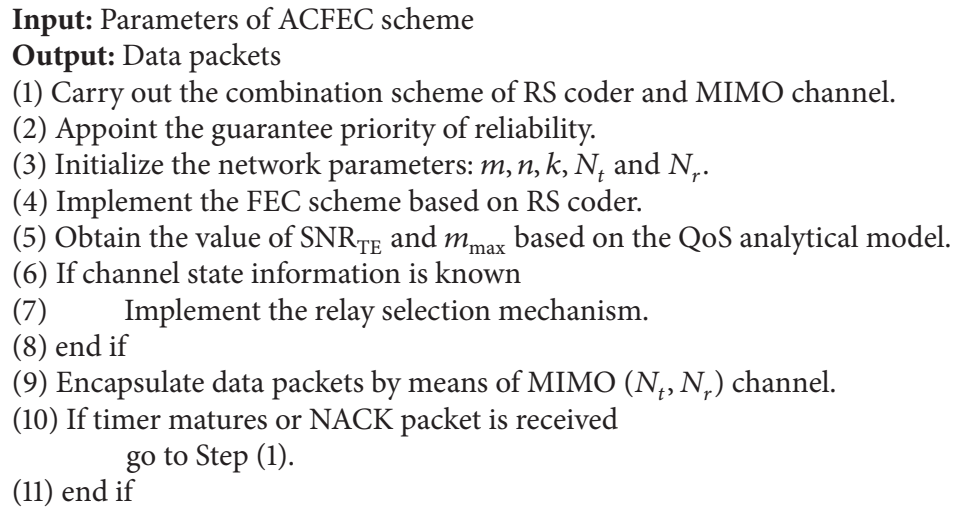

Algorithm 1: Algorithm of ACFEC at sender node.

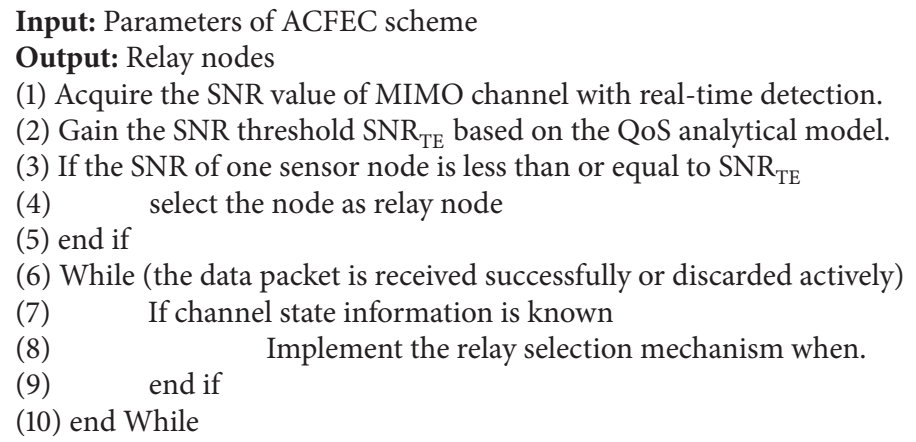

Algorithm 2: Relay selection algorithm of ACFEC at relay node.

Step (7): Steps (3) and (4) are implemented repeatedly until the data packet is received successfully or discarded actively.

The pseudocode for the proposed scheme at relay nodes is summarized in Algorithm 2.

\section{Receiver Node}

Step (8): checksum testing and RS encoding are implemented.

Step (9): if the result obtained is right, the data packet is accepted and ACK packet is sent simultaneously; otherwise, it is rejected and NACK packet is sent at the same time.

Step (10): deliver the correct data packet to the upper layer.

The pseudocode for the proposed scheme at receiver node is summarized in Algorithm 3.

\section{Performance Evaluation}

In this work, on the basis of the literature [13], we use NS-2 and VC++6.0 to simulate, analyze, and evaluate the performance of ordinary data transmission and multimedia communication using ACFEC, compared with no RS coder, RS coder alone, MIMO channel technology alone, combination of RS coder and MIMO through two group experiments. The experimental data is the average value after 100-time simulation and mathematical analysis.

4.1. Parameter Settings of Simulation and Mathematics. In experiment 1 , there are 50 sensor nodes, which move in a $1000 \mathrm{~m} \times 1000 \mathrm{~m}$ rectangular region. The mobility model is the random waypoint model. Each sensor node moves randomly at a speed uniformly, which is a random number of the interval $[0 \mathrm{~m} / \mathrm{s}, 20 \mathrm{~m} / \mathrm{s}]$. The MAC protocol is IEEE 802.15.4 protocol. The more detailed parameter settings are illustrated in Table 2.

For multimedia traffic of experiment 2, we use a medium quality MPEG4 video clip from the movie forman_qcif.yuv [14], which consists of 400 video frames. The structure of the group of picture is IBBPBBPBBPBB. The video frame rate is 25 frames per second.

4.2. Experiment Results and Discussion. Two case studies are designed and conducted, with the variation of SNR and simulation time, respectively. On one hand, Figure 5 shows 


Input: Data packets
Output: ACK or NACK pakcets
(1) Test Checksum.
(2) Implement RS encoding.
(3) If the result obtained is right
(4) Send ACK packet
(5) else if
(6) $\quad$ Rejecte the packet
(7)
(8) end if $\quad$ Send NACK packet
(9) Deliver the correct data packet to the upper layer.

Algorithm 3: Algorithm of ACFEC at receiver node.

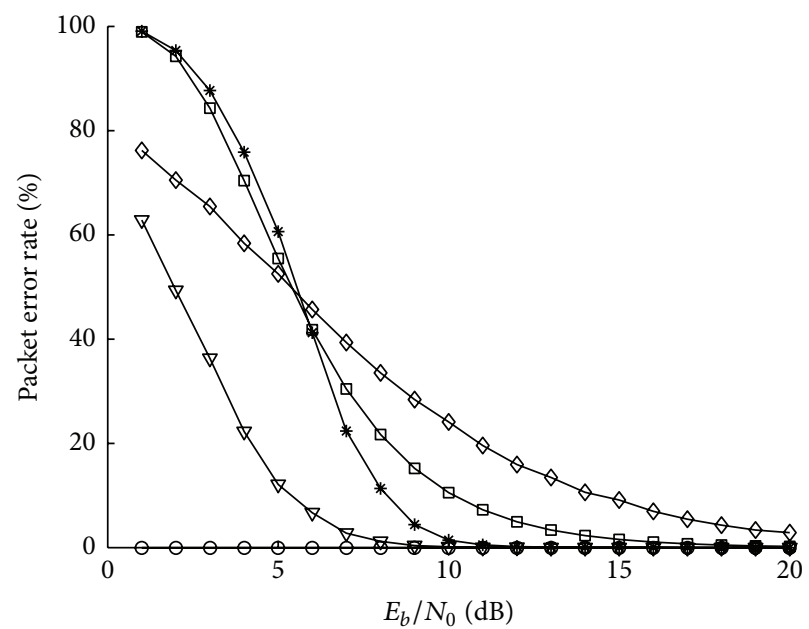

(a) Packet error rate

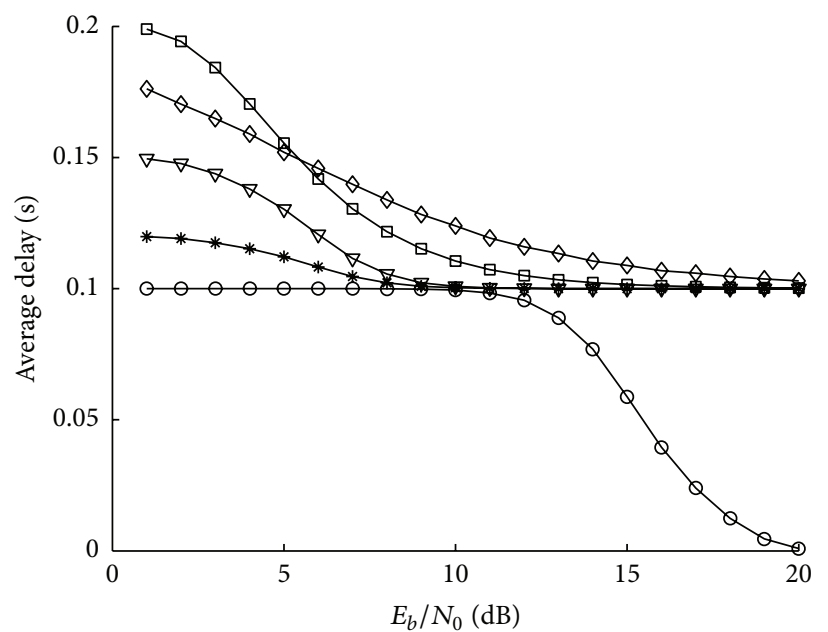

(b) Average delay

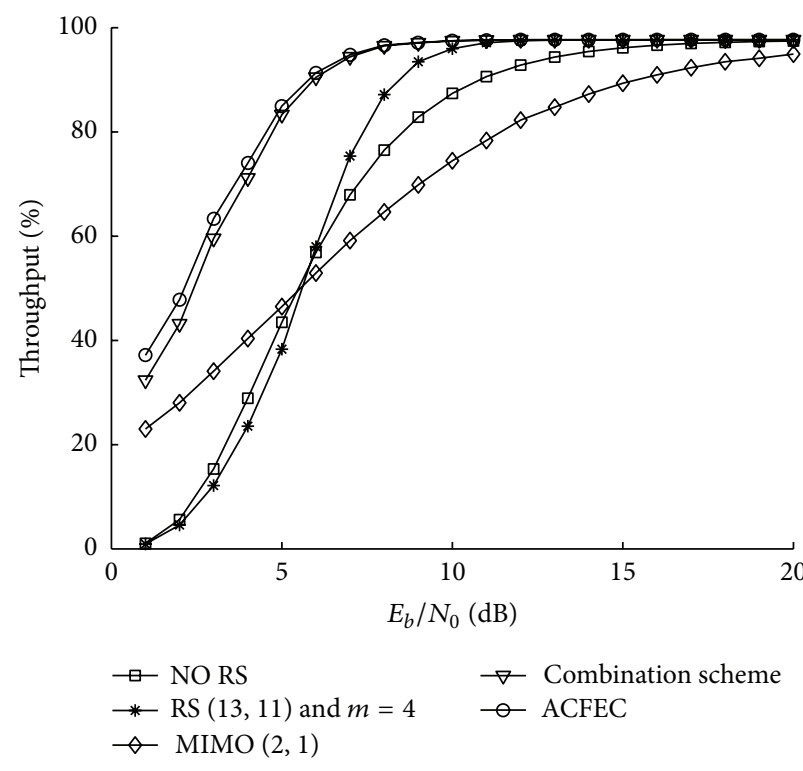

(c) Throughput

FIGURE 5: Performance with varying SNR. 


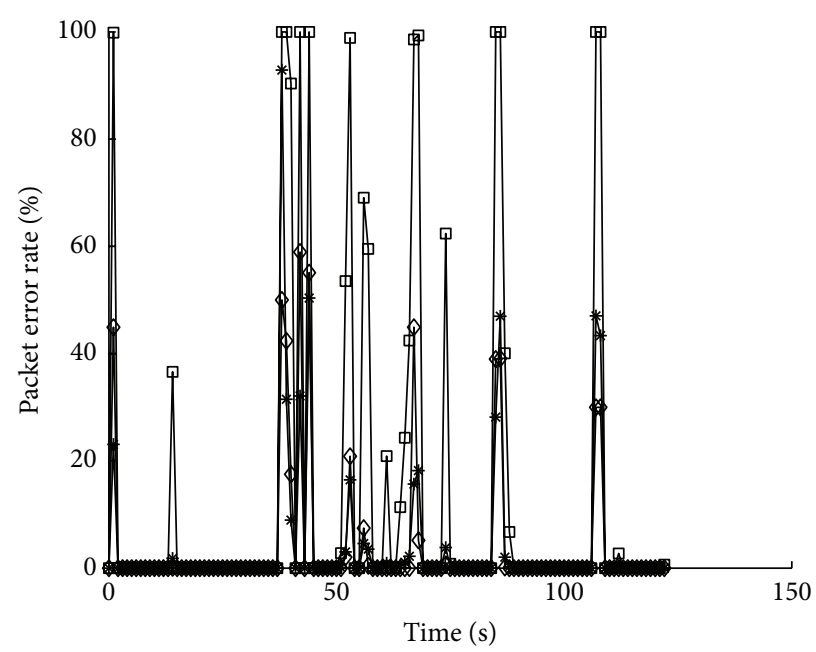

(a) Packet error rate

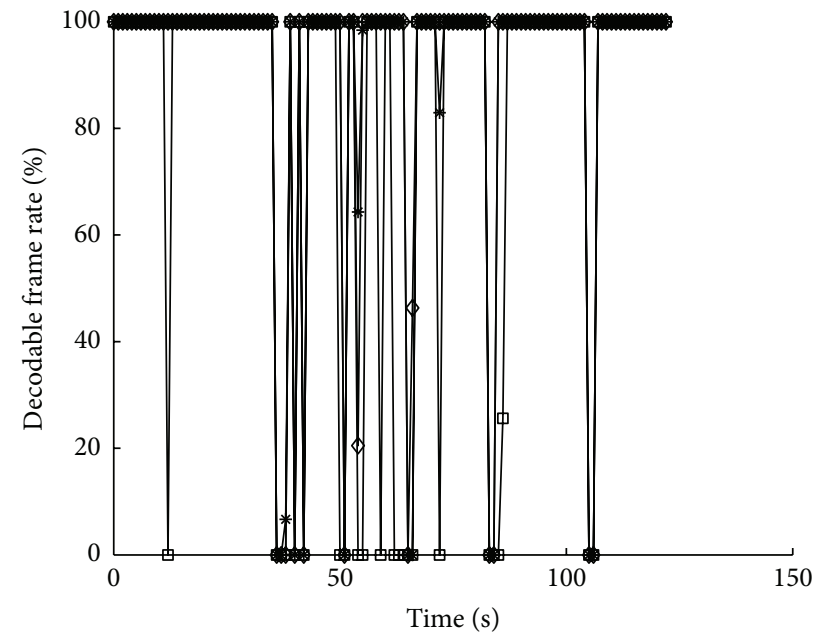

(b) Decodable frame rate

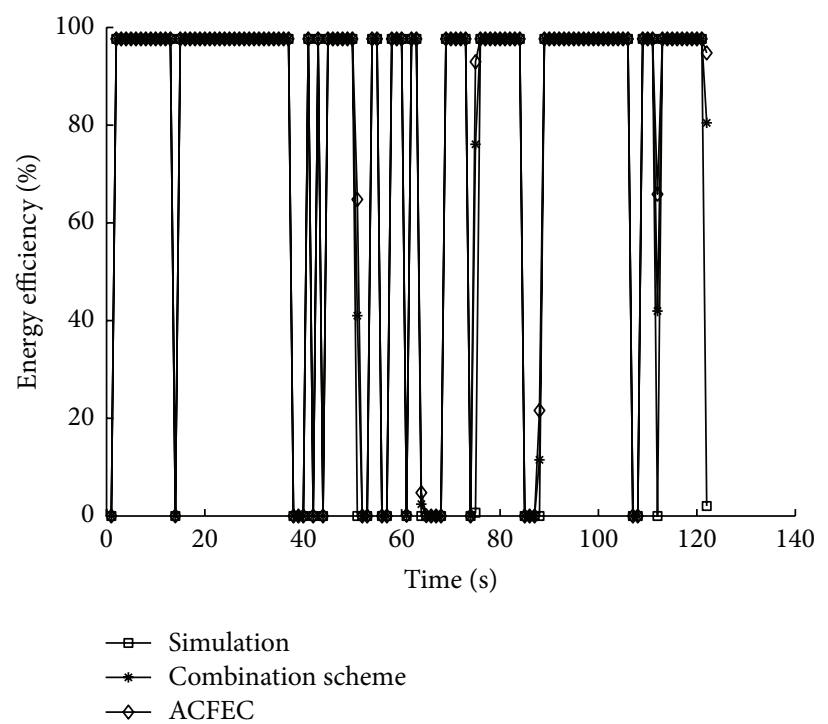

(c) Energy efficiency

Figure 6: Performance with multimedia traffic.

TABle 2: Parameter settings in experiment 1.

\begin{tabular}{lcc}
\hline Parameters & Definition & Value \\
\hline$B_{N}$ & Noise bandwidth & $30 \mathrm{kHz}$ \\
$R_{\text {radio }}$ & Data rate of CC1000 & $38.4 \mathrm{kbaud}$ \\
$T$ & Round trip time of one frame transmission & 0.01 second \\
\hline
\end{tabular}

three performance metrics of no RS coder, RS coder alone, MIMO channel technology alone, combination scheme of RS coder and MIMO (RS $(15,11) \& m=4$ and $\operatorname{MIMO}(2,1))$, and ACFEC as a function of SNR in experiment 1.

The result indicates that channel state has a significant impact on quality of data transmission in wireless sensor networks. We can observe tremendous improvement of performance with ACFEC, compared with the other four schemes. The throughput and energy efficiency of no RS coder, RS coder alone, and MIMO channel alone reduce and are close to zero gradually. That means that combination of RS coder and MIMO channel is superior to the alone scheme. In particular, even at a lower SNR of wireless channel, the quality of ACFEC remains in a good condition. The reasons are that not only the adaptive RS coder algorithm minimizes the average delay, but also the adaptive MIMO channel technology improves the energy efficiency; as a result, the network throughput ratio utilization increased.

On the other hand, Figure 6 shows three performance metrics of the static combination scheme of RS coder and MIMO channel (RS $(15,11) \& m=4$ and $\operatorname{MIMO}(2,1))$, as well as ACFEC in experiment 2. Figure 6(a) provides packet error rate for each video frame in different mechanisms. It is obvious that ACFEC provides the most reliable transmission. When using ACFEC, the packet error rate fluctuates little, and the average is the highest. These adaptive schemes in ACFEC are not only able to achieve higher decodable frame rate but also to improve the stability of the video transmission. 
Figure 6(b) shows the result of the decodable frame rate. As the transmission rate of multimedia data increases, the collision probability of data packets transmission increases significantly, leading to an unstable and dynamic decreasing tendency of decodable frame rate. The result demonstrates tremendous improvement of decodable frame rate with ACFEC as the static combination strategy. On this basis, we determine that ACFEC can primely accommodate the poor and dynamic wireless sensor network environment. This evident improvement depends on their stable energyefficiency-aware relay selection scheme based on SNR and adaptive MIMO channel strategy.

Figure 6(c) shows the energy utilization efficiency of different mechanisms. In wireless sensor networks, sensor nodes consume most energy for data transmission and reception, as well as error control. Obviously, the enhancement of data exchange gain and adaptive FEC can greatly reduce energy consumption for data transmission and retransmission. Comparing with other scheme, ACFEC achieves significant improvement in energy utilization efficiency.

\section{Conclusions}

Supporting diversity application over wireless sensor networks is more challenging due to the characteristics of wireless transmission. In this paper, an adaptive cooperative forward error correction (ACFEC) mechanism was proposed for satisfying the requirement.

Main contributions are as follows. First, considering the characteristics of FEC based on RS coder algorithm, we introduce a Markov chain model to study the QoS in wireless sensor networks. Second, we present energy-efficiencyaware adaptive relay selection algorithm based on SNR to reduce the ratio of potential damaged or lost opportunities. The purpose is to achieve higher system throughput and lower delay. Finally, we implement the ACFEC and carry out extensive evaluation. The results of mathematics and simulation demonstrate that ACFEC greatly improves the data transmission quality and achieves significant gains in terms of throughput and energy efficiency. As a result, we determine that the proposed mechanism is feasible for data communication in wireless sensor network.

\section{Acknowledgments}

This work is supported in part by the National Natural Science Foundation of China under Grant no. 61073197 and Scientific \& Technological Support Project of Jiangsu Province under Grant no. BE2011186.

\section{References}

[1] J. Yick, B. Mukherjee, and D. Ghosal, "Wireless sensor network survey," Computer Networks, vol. 52, no. 12, pp. 2292-2330, 2008.

[2] I. F. Akyildiz, T. Melodia, and K. R. Chowdury, "Wireless multimedia sensor networks: a survey," IEEE Wireless Communications, vol. 14, no. 6, pp. 32-39, 2007.

[3] J. S. Ahn, S. W. Hong, and J. Heidemann, "An adaptive FEC code control algorithm for mobile wireless sensor networks," Journal of Communications and Networks, vol. 7, no. 4, pp. 489-498, 2005.

[4] Y. Jin and G. W. Bai, "A cooperative FEC based on the model of GM, $(1,1)$ and IPv6 for wireless multimedia sensor networks," Journal of Convergence Information Technology, vol. 7, no. 18, pp. 230-239, 2012.

[5] G. Gupta, M. Misra, and K. Garg, "Energy efficient data gathering using prediction-based filtering in wireless sensor networks," International Journal of Information and Communication Technology, vol. 5, no. 1, pp. 75-94, 2013.

[6] S. K. Singh, M. P. Singh, and D. K. Singh, "Energy efficient transmission error recovery for wireless sensor networks," International Journal of Grid and Distributed Computing, vol. 3, no. 4, pp. 89-104, 2010.

[7] J. Vidhya and P. Dananjayan, "Cooperative MIMO transmissions in WSN using threshold based MAC protocol," International Journal of Wireless \& Mobile Networks, vol. 2, no. 3, pp. 196-210, 2010.

[8] A. Del Coso, U. Spagnolini, and C. Ibars, "Cooperative distributed MIMO channels in wireless sensor networks," IEEE Journal on Selected Areas in Communications, vol. 25, no. 2, pp. 402-414, 2007.

[9] M. Watson, M. Luby, and L. Vicisano, Forward Error Correction (FEC) Building Block, RFC, 5052, IETF, August 2007.

[10] J. Lacan, V. Roca, J. Peltotalo, and S. Peltotalo, Reed-Solomon Forward Error Correction (FEC) Schemes, RFC, 5510, IETF, April 2009.

[11] G. N. Bravos, G. Efthymoglou, and A. G. Kanatas, "MIMObased and SISO multihop sensor networks: energy efficiency evaluation," in Proceedings of 3rd IEEE International Conference on Wireless and Mobile Computing, Networking and Communications (WiMob '07), pp. 13-18, October 2007.

[12] S. M. Rizwan, V. Khurana, and G. Taneja, "Reliability analysis of a hot standby industrial system," International Journal of Modelling and Simulation, vol. 30, no. 3, pp. 315-322, 2010.

[13] N. Zaeri and S. Habib, "Exploration of sensor technology under simulation and measurement approaches," International Journal of Information and Communication Technology, vol. 3, no. 2, pp. 116-130, 2011.

[14] "Video Trace Library," http://trace.eas.asu.edu/yuv. 

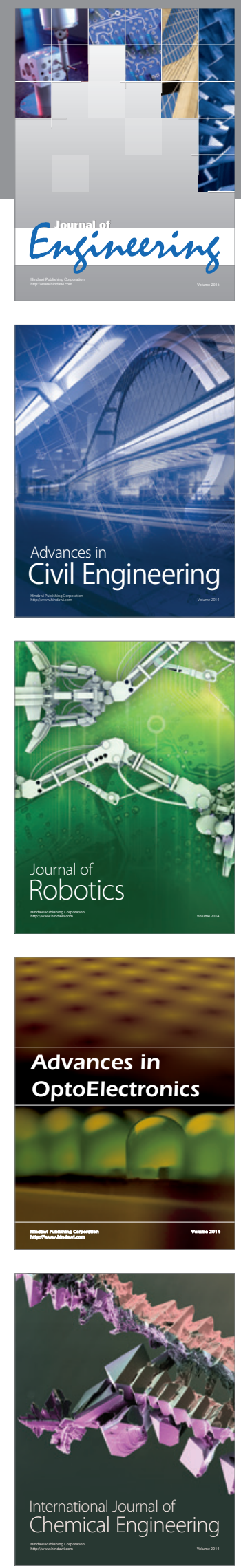

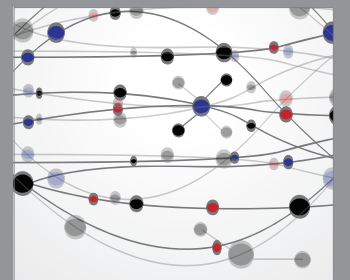

The Scientific World Journal
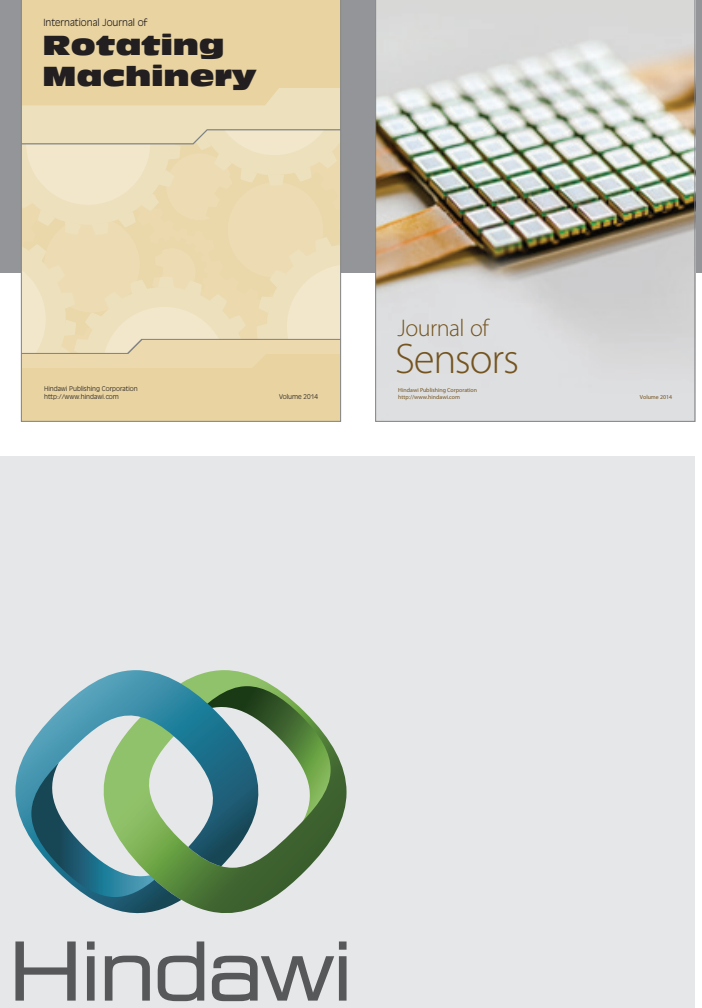

Submit your manuscripts at http://www.hindawi.com
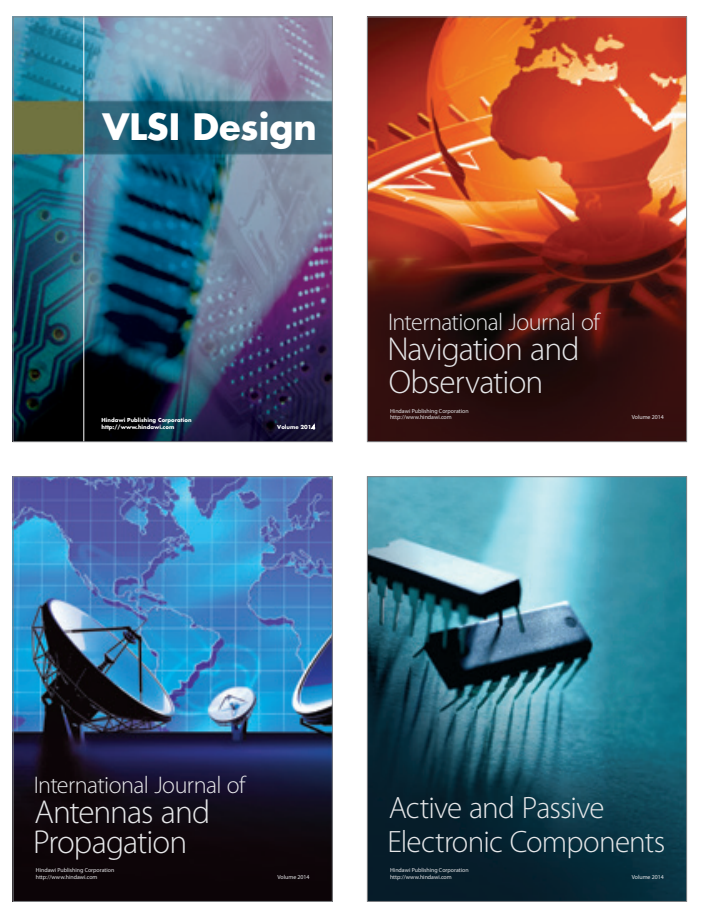
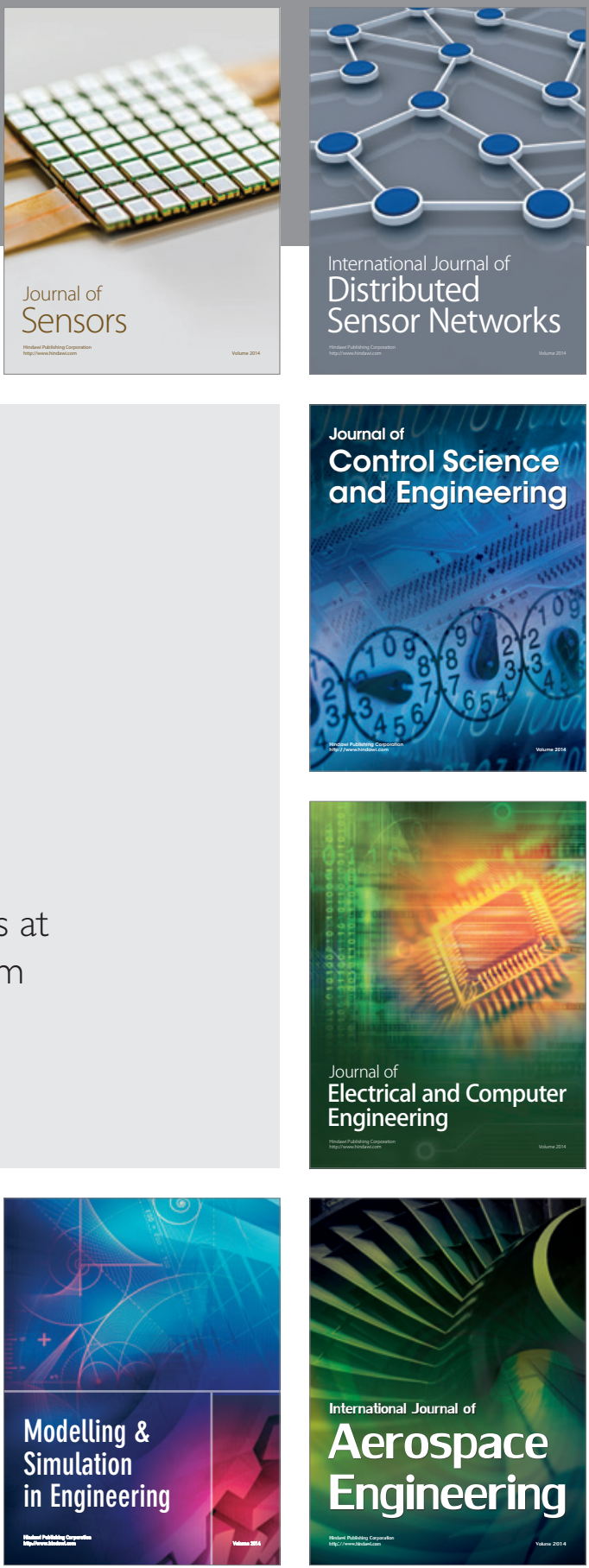

Journal of

Control Science

and Engineering
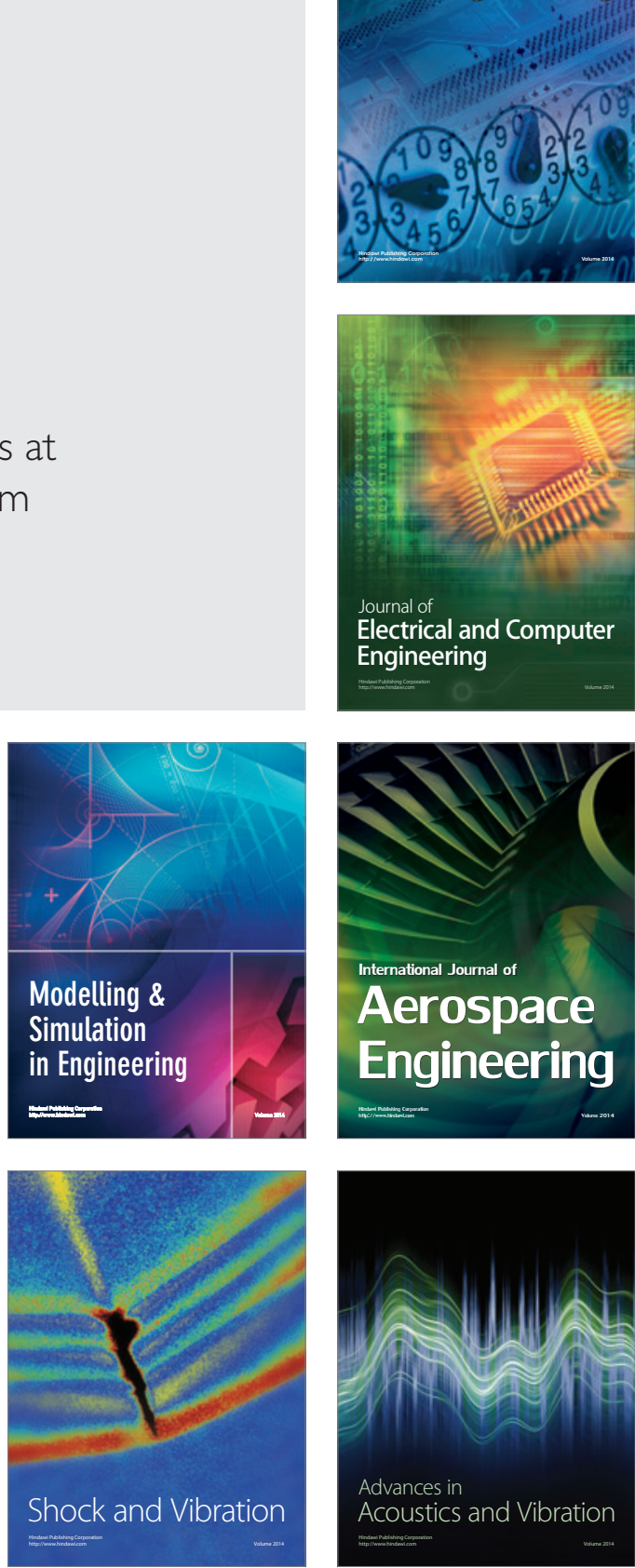